\title{
The mediating role of anthropomorphism in adopting the intentional stance towards humanoid robots
}

10 * Correspondence:

11 Corresponding Author

12 Prof. Agnieszka Wykowska

13 Agnieszka.Wykowska@iit.it

Abstract

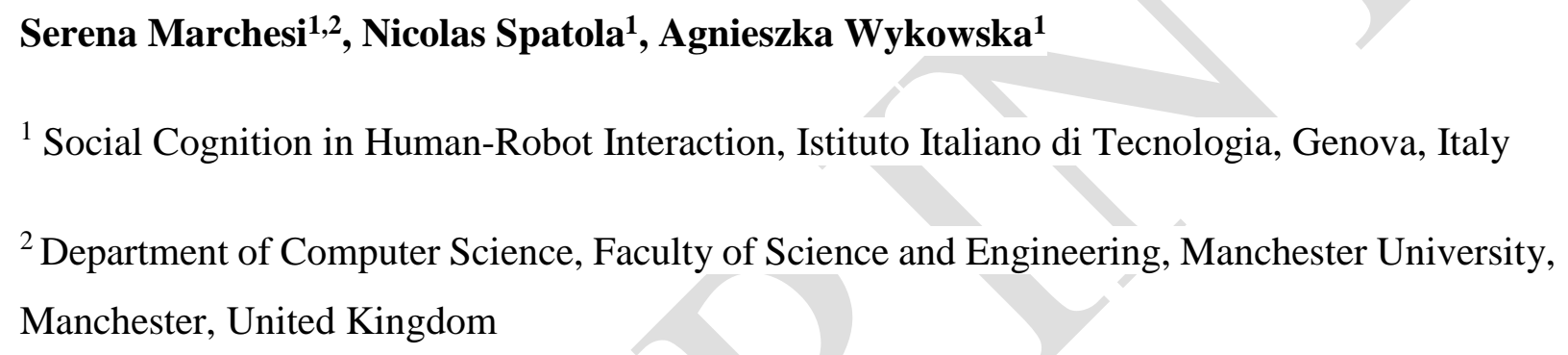

Keywords: Intentional Stance, Cross-Cultural Differences, Individual Differences, Human-Robot Interaction, Mediation

Evidence from cognitive psychology showed that cultural differences influence human social cognition, leading to a different activation of social cognitive mechanisms. A growing corpus of literature in Human-Robot Interaction is investigating how culture shapes cognitive processes like anthropomorphism or mind attribution when humans face artificial agents, such as robots. The present 
paper aims at disentangling the relationship between cultural values, anthropomorphism, and intentionality attribution to robots, in the context of the intentional stance theory. We administered a battery of tests to 600 participants from various nations worldwide and modeled our data with a path model. Results showed a consistent direct influence of collectivism on anthropomorphism but not on the adoption of the intentional stance. Therefore, we further explored this result with a mediation analysis that revealed anthropomorphism as a true mediator between collectivism and the adoption of the intentional stance. We conclude that our findings extend previous literature by showing that the adoption of the intentional stance towards humanoid robots depends on anthropomorphic attribution in the context of cultural values.

\section{Introduction}

There is an increasing trend to introduce robots in human' environments in daily contexts such as school, eldercare (for a review see Wykowska, 2020). As humans become exposed to robot presence, it is crucial to examine how robots are represented in the human mind, and how robot actions are perceived and interpreted. (for a review see Wykowska, 2021). Previous studies have shown that people differ in the degree of anthropomorphism [3], [4] and in the likelihood of attributing intentionality to robot actions (Marchesi et al., 2019; Bossi et al., 2020, Hegel et al., 2008; Thellman \& Ziemke, 2020). Apart from inter-individual differences such as personality traits [7]-[10], or, as reported by Ghiglino and Wykowska (2020), level of education, also cultural values might be a factor influencing the degree to which robots are perceived as humanlike. In the present study, we investigated how the cultural values of individualism and collectivism may influence the anthropomorphic representation of robots (i.e., the attribution of human characteristics to a non-human system) and consequently, the likelihood of adopting the intentional stance towards robots $[11]^{1}$. Cultural values such as individualism or collectivism are defined as the core norms and principles shared by a community to organize social life [12]) The importance of cultural values

2

${ }^{1}$ The concept of intentional stance has been introduced by Dennett (1989) to denote the strategy that people use to explain behaviour of others in intentional terms, that is, by reference to mental states. For example, when observing someone grasping a glass of water, one might explain this behaviour as "reaching for water, because s/he wants to drink". $\mathrm{S} /$ he "wants" is a mental state assumed to underlie the observed behaviour. For more elaborate description, see par. 1.3.

This is a provisional file, not the final typeset article 
Cultural values influence our general attitudes towards others and social exclusion/inclusion behaviours. For example, Varnum and colleagues [13] report that people from Eastern countries adopt a more holistic approach and are more prone to interdependency (i.e. the tendency to value more relational harmony rather than the individual [14]) compared to people from Western countries. Indeed, several authors reported differences in collectivistic and individualistic values between Eastern and Western countries, leading to the conclusion that collectivism and individualism are "Cultural Syndromes" (for a review see Trandis, 1993). According to Trandis (1993), a Cultural Syndrome is defined when constructs (such as collectivism/individualism) are: 1 - organized around a theme, a value; 2 - the differences within-culture are smaller than across cultures and 3- these constructs are clustered in line with geographic regions (not necessary countries). Therefore, individualism and collectivism can coexist in the same country. Cultural Syndromes are internalized by individuals, thus each person has an individual tendency to adhere to collectivism or individualism and this individual tendency influences our choices in everyday life, especially when we socially interact with other humans and shape the way we present ourselves to others (Bandura, 2002; Markus \& Kitayama, 1991; Vygogtskji, 1979).

\subsection{Anthropomorphism}

A second pivotal concept in our study is anthropomorphism in the definition given by Epley and colleagues (2007). The authors describe anthropomorphism as the attribution of human physical or mental characteristics to non-human agents (of any kind or form). Anthropomorphism can be elicited by three factors: 1- the availability of physical characteristics of the agent that can activate knowledge and heuristics related to humans; 2- the possibility of fulfilling the human need for connection and sociality; 3- individual traits [18]. While the first two factors depend on the agent and how much it activates the "human model" [19], the third relies on each individual, and researchers showed that different personality phenotypes have different tendencies to anthropomorphize [7].

\subsection{The intentional stance}

The third concept core to the present work is the concept of intentional stance. Dennett (1989, 2009) defines the intentional stance as the strategy humans spontaneously adopt when interpreting the 
behaviors of other agents with reference to mental states (she desires, he wishes, etc.). Interestingly, according to Dennett, this process takes place towards all agents that fulfill the epistemological requirement of being treated as rational. When this latter requirement is not met, humans can switch to the adoption of a different stance that allows a better explanation of the agent behavior, such as the design stance, defined as interpreting the agent's behavior with reference to how it is designed to behave. Several authors showed that the adoption of intentional stance can take place towards biological and non-biological agents [20]-[22]. As adoption of intentional stance relies on the attribution of human traits (mental states) to agents, it can be considered one aspect of a broader construct of anthropomorphism [8], [23]-[25].

\subsection{Cultural differences, anthropomorphism, and the adoption of intentional stance in human- robot interaction (HRI)}

Recent literature in HRI investigated the influences of cultural differences when humans face artificial agents (for a review see [26]. The cultural background seems to influence HRI regarding several features of social interaction: likeability, trust, and engagement [27], proxemics [28], recognition of facial expressions [29], and preferred style of communication [30]. On the one hand, findings from social psychology show a higher in-group bias for cultures with high collectivist values (Y. R. Chen et al., 2002; Yamagishi et al., 1998). For instance, Kuchenbrandt and colleagues reported that in-group bias influence people's evaluation of robots [31]. That is because robots are not members of the human (in-) group, and thus, collectivist cultures could be less prone to anthropomorphize them. In addition, Kovačić found that individualistic cultures present a more positive attitude toward technology (compared to collectivist cultures). Kovačić argues that individuals perceive technology as a potentially useful tool to help them perform better [32]. As positive attitudes tend to result in a more positive evaluation, the consequence could be the attribution of positive (human-like) characteristics such as warmth or sociability [33], [34]. Nevertheless, to the best of our knowledge, no study empirically investigated how individual cultural values and specifically, the individual tendency towards collectivism/individualism, shapes the adoption of the intentional stance towards a humanoid robot or anthropomorphism (and a fortiori the interaction between intentional stance and anthropomorphism). 
Recently, researchers have investigated whether the intentional stance can be adopted towards humanoid robots [38]-[40] (see also [41] for a review). Marchesi et al. (2019) created the InStance

110 Test (IST), a novel tool to assess the adoption of the intentional stance. The IST consists of a set of 111 scenarios, depicting the iCub humanoid robot [42] involved in several activities (Fig.1). Below each 112 scenario, two sentences are displayed as possible descriptions of the scenario. One description 113 addresses the scenario with reference to a mental state (i.e., intentional stance) and the other with 114 reference to a mechanistic explanation (i.e., design stance). Marchesi and colleagues asked participants 115 to choose which sentence fits best as a description of the scenario by moving a button along a slider.

116 The authors report that indeed there are individual differences in the adoption of the intentional stance, 117 which called for a deeper investigation of the relation between the individual likelihood to adopt the 118 intentional stance towards a robot and the individual tendency to anthropomorphize non-human 119 systems. Indeed, Marchesi et al. (2021) report that the individual differences in anthropomorphism are 120 associated with different levels of individual tendency to adopt the intentional stance towards robots 121 (a higher tendency to anthropomorphize is associated with a higher tendency to adopt the intentional 122 stance to explain the behavior of a humanoid robot) [43].

\subsection{Aim of the present research}

State-of-the-art research suggests that humans do adopt the intentional stance towards humanoid robots under some circumstances. Moreover, individual traits and other factors might differentiate individuals regarding their likelihood of adopting the intentional stance. Other biases and heuristics such as our tendency to anthropomorphize and individual cultural values such as collectivistic or individualistic attitudes might influence the individual differences. As differences in the level of individualism/collectivism can lead to a higher/lower tendency to anthropomorphize artificial agents . the present paper aims to disentangle the relationship between culture, the various dimensions of anthropomorphism, and the adoption of the intentional stance towards humanoid robots.

133 To address the aims of our study, we asked participants to complete a battery of questionnaires 134 to model the relationship between the above-mentioned constructs: Cultural Values Scale (CVSCALE, 135 [44], [45]) to measure individual adherence to cultural values; the Human-Robot Interaction scale 136 (HRIES, [46]) to measure anthropomorphism; and the InStance Test (IST, [39]) to measure the degree 
137 of adopting the intentional stance. Our hypotheses were two-folded: first, cultural values should

138 influence anthropomorphism [47][48] [49], and second, building on the results of Marchesi et al 139 (2021), anthropomorphism should positively mediate the causal relationship between the individual 140 tendency towards collectivism and the adoption of the intentional stance.

141

142

1432 Methods and measures

\section{$144 \quad 2.1 \quad$ Participants}

145 Six hundred - and - one participants from Singapore/Malaysia; the United States of America

146 (USA), the United Kingdom (UK); Germany, Spain, and Italy have been recruited from Prolific. com.

147 We selected these countries to access different levels of individualism-collectivism values and

148 therefore to reach a representative sample (see table 1 for demographics of each country) in return for

149 a payment of $3.3 £$. The study was approved by the local Ethical Committee (Comitato Etico Regione

150 Liguria) and was conducted in accordance with the Code of Ethics of the World Medical Association

151 (Declaration of Helsinki). Each participant provided written informed consent before taking part in the

152 experiment by clicking on the "accept" button at the beginning of the survey. All participants were 153 naïve to the purpose of this experiment.

154

155 Table 1 Demographic description of the sample

\begin{tabular}{|c|c|c|c|c|c|c|}
\hline Country & $\mathbf{n}$ & Male & Female & Non-Binary & $\begin{array}{l}\text { Prefer Not } \\
\text { to Answer }\end{array}$ & $\mathbf{M}_{\text {age }}$ \\
\hline USA & 100 & 56 & 42 & 2 & & 36.5 \\
\hline UK & 100 & 31 & 70 & & & 38.4 \\
\hline Italy & 100 & 54 & 42 & 4 & & 25.8 \\
\hline Spain & 100 & 71 & 27 & 1 & 1 & 27.7 \\
\hline Germany & 100 & 68 & 29 & 2 & 1 & 31.0 \\
\hline Singapore/Malaysia & 101 & 32 & 68 & 1 & & 27.2 \\
\hline
\end{tabular}


Participants completed a series of questionnaires tailored to assess different variables that according to our hypothesis, may influence the adoption of the intentional stance toward a humanoid robot. The order of presentation was as follows: Cultural Values Scale, InStance Test, and the HumanRobot Interaction Scale. All questionnaires were administered in English. To guarantee that the respondents would have sufficient knowledge of the English language to understand the task, and the items of the questionnaires, we administered the LexTALE test [50], which provides a measure of the individual level of English proficiency.

Cultural values measure. Hofstede (2011) developed a framework to describe cultural values with a five-dimension model that encompasses power distance, collectivism, uncertainty avoidance, masculinity vs. femininity, and long-term orientation vs. short-term orientation. Power distance refers to how much individuals accept and expect that power is distributed unevenly. Collectivism refers to the extent to which people rely on themselves (individualism) or their group (collectivism). Uncertainty avoidance refers to how much tolerance individuals have for ambiguity. Masculinity vs. femininity refers to how the inequalities between genders are perceived in society and how individuals internalize these inequalities. Finally, long-term orientation refers to the temporal orientation of a society, meaning to what extent individuals feel pressured to plan their life with a long-term mindset.

For the purposes of our study, we adopted the collectivism subscale of the Cultural Values Scale (CVSCALE,[45]), which has proven to be reliable in measuring Hofstede's dimensions of collectivism/individualism at the individual level ${ }^{2}$ (6 items, e.g., Individuals should sacrifice their selfinterest for the group; $\left.\omega=.860, \mathrm{CI}_{95 \%}[.843, .877]\right)$. In addition, as Hofstede posited a dimension named "indulgence", which addressed the extent to which a society values leisure time, moral discipline, happiness, and well-being (Enkh-Amgalan, 2016). Khans and Cox reported that indulgent cultures tend

7

${ }^{2}$ We administered and analyzed also the other subscales to control for covariance. The other subscales of the CVSCALE dimensions are power distance (6 items, e.g. People in higher positions should make most decisions without consulting people in lower positions; $\left.\omega=.816, \mathrm{CI}_{95 \%}[.793, .839]\right)$, uncertainty avoidance (5 items, e.g. It is important to closely follow instructions and procedures; $\left.\omega=.862, \mathrm{CI}_{95 \%}[.845, .880]\right)$, long-term orientation (6 items, e.g., Long-term planning is important; $\omega=.749, \mathrm{CI}_{95 \%}[.719, .780]$ ), and masculinity (4 items, e.g. It is more important for men to have a professional career than it is for women; $\left.\omega=.831, \mathrm{CI}_{95 \%}[.808, .853]\right)$. 
182 to create new technology as a way to improve life, to value the expression of emotions and freedom of 183 speech. To explore the indulgence dimension, we developed 5 items added at the very last of the

184 CVSCALE questionnaire (e.g., Freedom of speech is important (see supplementary materials for the 185 complete list of items); $\omega=.715, \mathrm{CI}_{95 \%}[.679, .751]$ ) [51], [52]. For each item participants had to 186 indicate the extent to which they agree or disagree with the statement from 1 "Disagree strongly" to 7 187 “Agree strongly”.

Table 2. Descriptive Statistics of the filtered sample for the collectivism subscale (Yoo et al., 2011)

\begin{tabular}{ccccccc}
\hline & \multicolumn{7}{c}{ COLLECTIVISM } \\
\cline { 2 - 7 } & Ger & Ita & Singa_Malay & Spain & UK & USA \\
\hline Valid & 95 & 96 & 95 & 94 & 98 & 94 \\
Mean & 4.248 & 4.115 & 4.394 & 4.723 & 4.296 & 4.314 \\
Std. Deviation & 1.006 & 1.064 & 1.024 & 0.934 & 1.120 & 1.281 \\
\hline
\end{tabular}

Anthropomorphism measure. Spatola and colleagues (2020), created the Human-Robot

190 Interaction Evaluation Scale (HRIES) to assess the individual tendency to anthropomorphize social 191 robots. The authors report four subscales: Sociability (i.e., to what extent the robot is perceived as 192 social, $\omega=.840, \mathrm{CI}_{95 \%}[.811, .865]$ ), Agency (i.e., to what extent the robot it perceived to have agency 193 in its environment, $\omega=.767, \mathrm{CI}_{95 \%}[.727, .803]$ ), Animacy (i.e., to what extent the robot is perceived as 194 having smooth movements, $\omega=.807$, $\mathrm{CI}_{95 \%}[.778, .833]$ ), and Disturbing (i.e., to what extent the robot 195 is perceived as uncanny, $\omega=.883, \mathrm{CI} 95 \%[.863, .900])$. Participants were asked to evaluate how closely 196 a series of adjectives would describe the robot (i.e., "Warm", "Alive", "Real"). The items were 197 evaluated on a 7-point Likert scale (1: "Not at all”, 7: “Totally").

Table 3a. Descriptive Statistics of the filtered sample for the HRIES (Spatola et al., 2020)

\begin{tabular}{|c|c|c|c|c|c|c|c|c|c|c|c|c|}
\hline & \multicolumn{6}{|c|}{ HRIES_SOCIABILITY } & \multicolumn{6}{|c|}{ HRIES_ANIMACY } \\
\hline & Ger & Ita & Singa_M & Malay Spain & UK & USA & Ger & Ita & Singa_M & Malay Spain & UK & USA \\
\hline Valid & 95 & 96 & 95 & 94 & 98 & 94 & 95 & 96 & 95 & 94 & 98 & 94 \\
\hline Mean & 4.921 & 4.589 & 4.734 & 4.987 & 4.316 & 4.779 & 3.634 & 3.469 & 3.639 & 3.489 & 3.028 & 3.596 \\
\hline $\begin{array}{c}\text { Std. } \\
\text { Deviation }\end{array}$ & 1.100 & 1.046 & 0.963 & 0.859 & 1.471 & 1.103 & 1.092 & 1.213 & 1.356 & 1.274 & 1.367 & 1.363 \\
\hline
\end{tabular}

Table 3b. Descriptive Statistics of the filtered sample for the HRIES (Spatola et al., 2020)

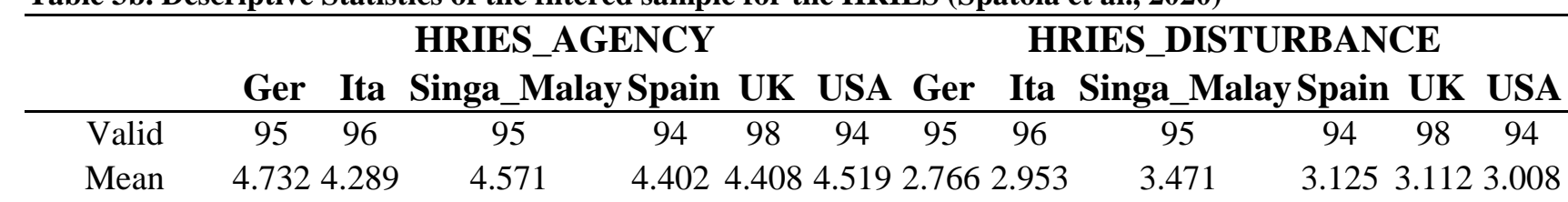

This is a provisional file, not the final typeset article 
Table 3b. Descriptive Statistics of the filtered sample for the HRIES (Spatola et al., 2020)

HRIES_AGENCY HRIES_DISTURBANCE

Ger Ita Singa_MalaySpain UK USA Ger Ita Singa_MalaySpain UK USA

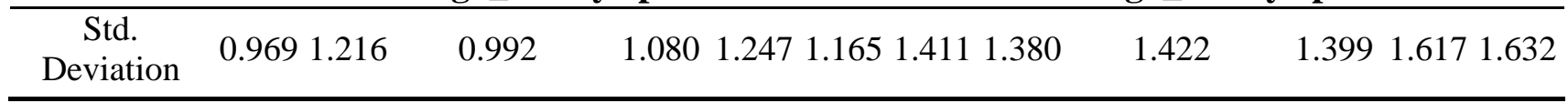
to assess the individual tendency of adopting the intentional stance towards a humanoid robot. Overall, it consists in 34 scenarios $\left(\omega=.880, \mathrm{C} \mathrm{I}_{95 \%}[.867, .894]\right)$, each one made of three static images depicting

204 the iCub robot (Metta et al., 2010). Below, two sentences are presented (one mentalistic, thus representing the adoption of the intentional stance, and the other mechanistic, thus representing the adoption of the design stance) as possible descriptions of each scenario (Fig.1). Participants' task is to

207 choose the sentence they consider a good fit for the depicted scenario by moving a button on a slider.

208 The obtained score ranges from 0 (totally mechanistic, i.e., design stance) to 100 (totally mentalistic,

209 i.e., intentional stance). For the complete list of items, refer to [39] Supplementary Materials.

210

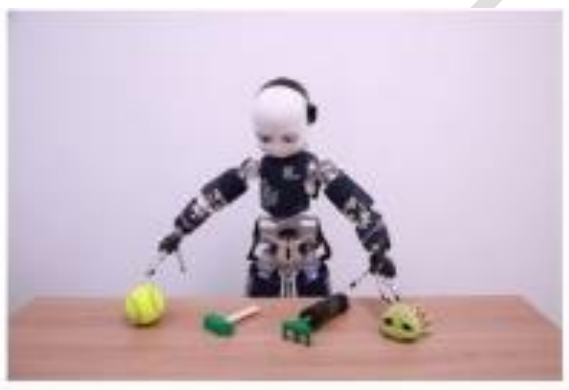

iCub adjusts the force to the weight of the object.

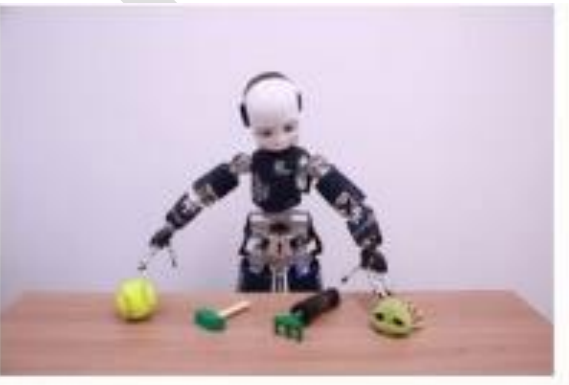

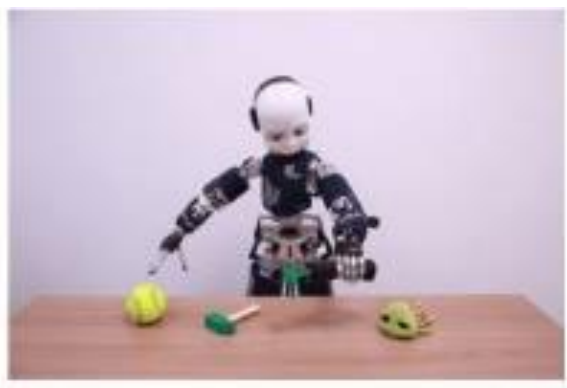

$i$ Cub pretends to be a gardener.

211 Figure 1: Example item from the IST (Marchesi et al., 2019)

Table 4. Descriptive Statistics of the filtered sample for the InStance Test (Marchesi et al., 2019)

\begin{tabular}{ccccccc}
\hline & \multicolumn{7}{c}{ INSTANCE TEST } \\
\cline { 2 - 7 } & Ger & Ita & Singa_Malay & Spain & UK & USA \\
\hline Valid & 95 & 96 & 95 & 94 & 98 & 94 \\
Mean & 36.672 & 36.005 & 43.365 & 37.827 & 32.445 & 37.923 \\
Std. Deviation & 14.825 & 16.775 & 13.910 & 15.790 & 17.382 & 17.430 \\
\hline
\end{tabular}




\section{Analyses and results}

\section{$215 \quad 3.1 \quad$ Filtering procedure}

Participants with missing values and values that resulted over or below 2.5 SD on the variable of interests were considered as outliers, leading to a total sample of $\mathrm{N}=572$ participants (Singapore/Malaysia $n=95$; USA $n=94 ;$ UK n = 98; Germany $n=95$; Spain $n=94$; Italy $n=96$ ). All the analyses were performed with R (version 4.0.2 [53]) and JASP v.0.14.1 (2020), using the SEM package.

\subsection{Comparison across Countries on IST and HRIES subscales}

To explore differences in the collectivistic tendency, in the adoption of the intentional stance and in the tendency to anthropomorphize across Countries, we performed non-parametrical Kruskal-Wallis test on the collectivism, IST and HRIES subscales scores. Results showed a significant difference in the collectivism subscale of the CVSCALE $\left[\chi^{2}=16.84, \mathrm{df}=5, \mathrm{p}=.004, \varepsilon^{2}=.029\right]$ and in the IST $\left[\chi^{2}\right.$ $\left.=21.42, \mathrm{df}=5, \mathrm{p}<.001, \varepsilon^{2}=.037\right]$. Moreover, statistical significance emerged also for the Sociability subscale $\left[\chi^{2}=17.11, \mathrm{df}=5, \mathrm{p}=.004, \varepsilon^{2}=.029\right]$, Animacy subscale $\left[\chi^{2}=15.52\right.$, df $=5, \mathrm{p}=.008, \varepsilon^{2}=$ $.027]$ and Disturbance subscale $\left[\chi^{2}=12.69, \mathrm{df}=5, \mathrm{p}=.026, \varepsilon^{2}=.022\right]$ of the HRIES. No statistical significant effect emerged for the Agency subscale of the HRIES $\left[\chi^{2}=8.56, \mathrm{df}=5, \mathrm{p}=.127, \varepsilon^{2}=.014\right]$.

Post-Hoc comparison with Tukey HSD correction are reported in the supplementary materials.

To further explore the relationship between collectivistic tendency, adoption of the intentional stance, and anthropomorphic attribution we performed a path model analysis.

\subsection{Structural Equation Model}

First, to explore the structural relationship between our variables, we conducted a path model analysis. We defined our model introducing all the subscales from the CVSCALE (to control for covariance) as first-order variables, all the subscales from the HRIES as second-order variables, and the IST as the outcome variable. We controlled for covariance between same-level variables. In 
240 addition, to account for possible differences in the knowledge of the English language, we added the 241 LexTALE test [50] as a covariate (see Supplementary Materials for all model parameters). All 95\%

242 Confidence Intervals were calculated with a bias-corrected percentile bootstrap analysis with 10,000 243 samples [54], with a Maximum Likelihood estimator, that has been proven to provide more precise 244 model fit indices and less biased parameters, compared to other estimators such as Generalized Least 245 Squares and Weighted Least Squares [55]. All variables were z-score corrected. The model fit indices 246 and Information Criteria are reported

247 respectively in Figure 2.

\subsection{Structural Equation Model Results}

Figure 2 represents all the significant paths that emerged from the path model, revealing a 251 consistent direct influence of collectivism on the anthropomorphism constructs (specifically on the 252 Sociability, Agency, and Animacy subscales) but not on the IST. Given our initial hypothesis, we 253 further explored the relationship indirect relationship between collectivism and IST (collectivism $\rightarrow$ 254 anthropomorphism $\rightarrow$ intentional stance) with a mediation model, considering the above-mentioned 255 variables as the predictor, mediator, and outcome, respectively. All paths' parameters are reported in 256 the supplementary materials. 


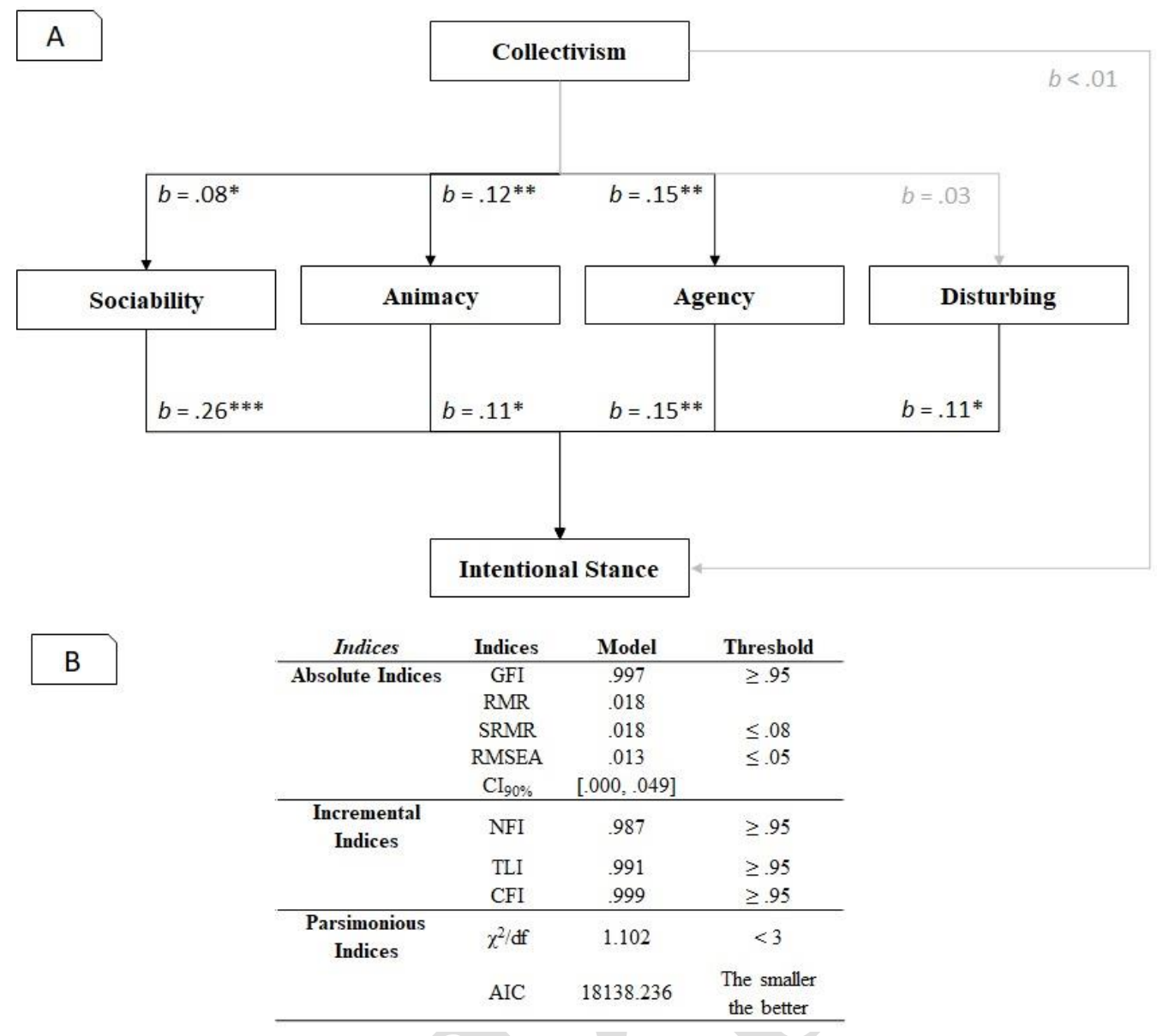

Figure 2. Panel A: Model path. We reported the significant (in black) and non-significant (in grey) paths with the associated $b$ statistics. Panel B: Fit indices of our model.

\subsection{Indirect effects}

As a second step, building upon the significant paths in the path model analysis, we tested the

263 mediating effect of anthropomorphism between the individual collectivism tendency and the adoption

264 of the intentional stance. As in the previous analysis, all data points were z-score corrected. The strategy to calculate the $95 \%$ Confidence Intervals was kept constant (a bias-corrected percentile bootstrap analysis with 10,000 samples), as the Maximum Likelihood estimator.

The indirect effects of collectivism on IST through the HRIES scale were significant when mediated by the Sociability subscale $[\mathrm{b}=0.025$, bootSE $=0.011, \mathrm{z}=2.253, \mathrm{p}=0.024$, bootC.I. $95 \%$ 

$95 \%(0.007 ; 0.047)]$. No indirect effect of collectivism on IST mediated by the Disturbing subscale (bootC.I. included 0, see Table 2) was observed.

Table 2 - Indirect effects

\begin{tabular}{|c|c|c|c|c|c|c|c|c|c|}
\hline & & & & \multirow[b]{2}{*}{ Estimate } & \multirow[b]{2}{*}{ Std. Error } & \multirow[b]{2}{*}{ z-value } & \multirow[b]{2}{*}{$\mathbf{p}$} & \multicolumn{2}{|c|}{$5 \%$ Confidence Interval } \\
\hline & & & & & & & & Lower & Upper \\
\hline$\overline{\text { CV_COLL }}$ & $\rightarrow$ & HRIES_SOC & $\rightarrow$ IST & 0.025 & 0.011 & 2.253 & 0.024 & 0.006 & 0.054 \\
\hline CV_COLL & $\rightarrow$ & HRIES_AN & $\rightarrow$ IST & 0.016 & 0.008 & 1.996 & 0.046 & 0.003 & 0.039 \\
\hline CV_COLL & $\rightarrow$ & HRIES_AG & $\rightarrow$ IST & 0.023 & 0.010 & 2.424 & 0.015 & 0.007 & 0.047 \\
\hline CV_COLL & $\rightarrow$ & HRIES_DIST & $\rightarrow$ IST & 0.001 & 0.005 & 0.280 & 0.780 & -0.009 & 0.015 \\
\hline
\end{tabular}

Note. Delta method standard errors, bias-corrected percentile bootstrap confidence intervals, ML estimator. NB: Not all bootstrap samples were successful: CI based on 9998 samples.

Following Zhao and colleagues' classification (Zhao et al., 2010), anthropomorphism seems to play the role of a true mediator between the individual collectivism tendency and the adoption of the intentional stance towards a humanoid robot. This seems particularly true for the mediation role of the perceived level of Sociability, Animacy, and Agency of the robot, while the Disturbing dimension seemed not to mediate the effect. Nonetheless, the total indirect effect of Collectivism on the IST is significant $[\mathrm{b}=0.065$, bootSE $=0.018, \mathrm{z}=3.623, \mathrm{p}=<.001$, bootC.I. $95 \%(0.029 ; 0.104)]$, indicating the total mediating role of attributed anthropomorphism on the relationship between the individual tendency towards collectivism and the individual likelihood of adopting the intentional stance towards a humanoid robot (Table 2). The higher the level of collectivism, the higher the anthropomorphic attribution, and the higher the likelihood of adopting the intentional stance towards a humanoid robot.

\subsection{Exploratory analyses}

In addition, to confirm that our model was indeed the best to describe the relationship between the constructs of interest, we post-hoc compared our mediation analysis with a second model that considered the adoption of the intentional stance as mediator (collectivism $\rightarrow$ intentional stance $\rightarrow$ anthropomorphism). Results showed that there is no mediation effect of the intentional stance on the 
293 This showed that the tendency towards collectivism influenced the tendency to anthropomorphize and, 294 only through anthropomorphism, collectivism influenced the tendency to adopt the intentional stance 295 towards humanoid robots.

\section{Discussion}

In the present paper, we aimed at disentangling the relationship between cultural values, the tendency to anthropomorphize, and the tendency to adopt the intentional stance towards a humanoid robot. To address this aim, we first built a structural model to test whether our hypothesized relationship between the variables (and relative sub-constructs) would hold: differences in cultural values should influence anthropomorphism and the adoption of the intentional stance. Results showed that cultural values, in particular the level of individualism/collectivism, directly influence anthropomorphism, but not the adoption of the intentional stance. The higher the collectivism, the higher the anthropomorphism. Our second step was to investigate whether anthropomorphism would play a mediating role between collectivism and the adoption of the intentional stance. The mediation results showed that when considering the role of anthropomorphism as a mediator between culture (as a predictor) and the adoption of the intentional stance towards robots (as the outcome), the relationship was significant. In particular, our results showed that the Agency, Sociability, and Animacy subscales of the HRIES were positive mediators between collectivism and adoption of the intentional stance. Interestingly, the "disturbing" subscale of HRIES (i.e. negative appraisal) seems to rely on a parallel 313 Stance. The higher the score on the disturbing subscale, the higher the IST. A tentative interpretation of this result could rely on the distinctiveness threat hypothesis discussed by Ferrari et al. (2016). The authors argue that individuals could feel threatened by the increased (imaginative) similarity of robots' appearance and capacities to humans [56]. In this context, the observer does not adopt a design stance but on the contrary, overestimates the abilities of the robot, attributing to the artificial agent humanlike characteristics such as intentions. In other words, the distinctiveness threat results in an overestimation of the human-like properties of the robot (as a negative expectation). This result is consistent with previous literature, where collectivistic cultures are reported to have more negative attitudes towards robots and do not perceive them as part of their in-group (Bartneck et al., 2007, Wang et al., 2010, Marchesi, Roselli and Wykowska, 2021). 
More generally, the model showed a strong influence of collectivism on anthropomorphism and

324 - through anthropomorphism - on the adoption of the intentional stance towards a humanoid robot.

325 The relationship in the reverse direction (intentional stance $\rightarrow$ anthropomorphism) was not observed.

326 In other words, cultural values, in particular collectivism, influence our perception of robots in terms

327 of anthropomorphic attributions. This result is in line with previous literature that shows how

328 collectivistic cultures tend to anthropomorphize, like more and trust more, anthropomorphically-

329 shaped or zoomorphic robots (for a review see Lim, Rooksby, and Cross, 2020). The present results

330 extend the previous findings by demonstrating that the adoption of the intentional stance towards robots

331 depends on anthropomorphism in a context of cultural values. Therefore, interpretation of the robot's

332 actions would depend on prior attribution of anthropomorphic characteristics. This attribution would

333 be influenced by the cultural values adopted by the observer.

Future experiments should attempt to create a vaster and more comprehensive theoretical model of how these variables and their facets interact with each other when humans predict the behaviour of a robot. Moreover, more theoretical and empirical investigations should explore the presence of new variables that may play a role in the adoption of the intentional stance towards humanoid robots.

\section{$5 \quad$ Author Contributions}

SM and AW designed the task. SM performed data collection, SM and NS performed data analysis, SM, NS, and AW discussed the data. SM, NS, and AW wrote and edited the manuscript. All authors contributed to reviewing the manuscript and approved it.

\section{$345 \quad 6 \quad$ Funding}

This work has received support from the European Research Council under the European

347 Union's Horizon 2020 research and innovation program, ERC Starting Grant, G.A. number: ERC348 2016-StG-715058, awarded to Agnieszka Wykowska. The content of this paper is the sole 349 responsibility of the authors. The European Commission or its services cannot be held responsible for any use that may be made of the information it contains. 


\section{$7 \quad$ References}

353

354

355

356

357

358

359

360

361

362

363

364

365

366

368

369

370

371

372

373

374

375

376

[1] A. Wykowska, "Social Robots to Test Flexibility of Human Social Cognition," Int. J. Soc. Robot., vol. 12, no. 6, pp. 1203-1211, 2020, doi: 10.1007/s12369-020-00674-5.

[2] A. Wykowska, "Robots as Mirrors of the Human Mind," Curr. Dir. Psychol. Sci., vol. 30, no. 1, pp. 34-40, 2021, doi: 10.1177/0963721420978609.

[3] H. Cullen, R. Kanai, B. Bahrami, and G. Rees, "Individual differences in anthropomorphic attributions and human brain structure," Soc. Cogn. Affect. Neurosci., vol. 9, no. 9, pp. 12761280, 2013, doi: 10.1093/scan/nst109.

[4] M. G. Chin, V. K. Sims, B. Clark, and G. R. Lopez, "Measuring Individual Differences in Anthropomorphism toward Machines and Animals," Proc. Hum. Factors Ergon. Soc. Annu. Meet., vol. 48, no. 11, pp. 1252-1255, 2004, doi: 10.1177/154193120404801110.

[5] F. Hegel, S. Krach, T. Kircher, B. Wrede, and G. Sagerer, "Understanding social robots: A user study on anthropomorphism," Proc. 17th IEEE Int. Symp. Robot Hum. Interact. Commun. ROMAN, pp. 574-579, 2008, doi: 10.1109/ROMAN.2008.4600728.

[6] S. Thellman and T. Ziemke, "Do You See what I See? Tracking the Perceptual Beliefs of Robots," iScience, vol. 23, no. 10, p. 101625, 2020, doi: 10.1016/j.isci.2020.101625.

[7] N. Spatola and A. Wykowska, "The personality of anthropomorphism: How the need for cognition and the need for closure define attitudes and anthropomorphic attributions toward robots," Comput. Human Behav., vol. 122, p. 106841, 2021, doi: 10.1016/j.chb.2021.106841.

[8] G. Airenti, "The development of anthropomorphism in interaction: Intersubjectivity, imagination, and theory of mind," Front. Psychol., vol. 9, no. NOV, pp. 1-13, 2018, doi: 10.3389/fpsyg.2018.02136.

[9] A. Waytz, J. Cacioppo, and N. Epley, "Who sees human? The stability and importance of individual differences in anthropomorphism," Perspect. Psychol. Sci., vol. 5, no. 3, pp. 219232, 2010, doi: 10.1177/1745691610369336.

This is a provisional file, not the final typeset article 
[10] A. D. Kaplan, T. Sanders, and P. A. Hancock, "The relationship between extroversion and the tendency to anthropomorphize robots: A Bayesian analysis," Front. Robot. AI, vol. 6, no. JAN, 2019, doi: 10.3389/frobt.2018.00135.

[11] J. Haugeland and D. C. Dennett, "True Believers:The Intentional Strategy and Why It Works," Mind Des. II, 2020, doi: 10.7551/mitpress/4626.003.0003.

[12] H. C. Triandis, Individualism and collectivism. 2018.

[13] M. E. W. Varnum, I. Grossmann, S. Kitayama, and R. E. Nisbett, "The origin of cultural differences in cognition: The social orientation hypothesis," Curr. Dir. Psychol. Sci., vol. 19, no. 1, pp. 9-13, 2010, doi: 10.1177/0963721409359301.

[14] S. Kitayama, M. Karasawa, K. B. Curhan, C. D. Ryff, and H. R. Markus, "Independence and interdependence predict health and wellbeing: Divergent patterns in the United States and Japan," Front. Psychol., vol. 1, no. SEP, pp. 1-10, 2010, doi: 10.3389/fpsyg.2010.00163.

[15] A. Bandura, "Social cognitive theory in cultural context," Appl. Psychol., vol. 51, no. 2, pp. 269-290, 2002, doi: 10.1111/1464-0597.00092.

[16] H. R. Markus and S. Kitayama, "17. Markus \& Kitayama (1991),” Psychol. Rev., vol. 98, no. 2, pp. 224-253, 1991.

[17] S. T. Parker, : Mind in Society: The Development of Higher Psychological Processes . L. S. Vygotsky, vol. 81, no. 4. 1979.

[18] N. Epley, A. Waytz, and J. T. Cacioppo, "On Seeing Human: A Three-Factor Theory of Anthropomorphism," Psychol. Rev., vol. 114, no. 4, pp. 864-886, 2007, doi: 10.1037/0033295X.114.4.864.

[19] E. Wiese, G. Metta, and A. Wykowska, "Robots as intentional agents: Using neuroscientific methods to make robots appear more social," Front. Psychol., vol. 8, no. OCT, pp. 1-19, 2017, doi: 10.3389/fpsyg.2017.01663.

[20] F. Happé and U. Frith, "Theory of Mind in Autism," Learn. Cogn. Autism, pp. 177-197, 1995, doi: 10.1007/978-1-4899-1286-2_10. 
403

404

405

406

407

408

409

410

411

412

413

414

415

416

417

418

419

420

421

422

423

424

425

426

427

428

429

[21] F. Heider and M. Simmel, "University of Illinois Press http://www.jstor.org/stable/1416950 .," Am. J. Psychol., vol. 57, no. 2, pp. 243-259, 1944.

[22] J. Zwickel, "Agency attribution and visuospatial perspective taking," Psychon. Bull. Rev., vol. 16, no. 6, pp. 1089-1093, 2009, doi: 10.3758/PBR.16.6.1089.

[23] M. Dacey and M. Dacey, "Anthropomorphism as Cognitive Bias Mike Dacey," no. 2002, pp. $1-19,1957$.

[24] A. Waytz, N. Epley, and J. T. Cacioppo, "Social cognition unbound: Insights into anthropomorphism and dehumanization," Curr. Dir. Psychol. Sci., vol. 19, no. 1, pp. 58-62, 2010, doi: 10.1177/0963721409359302.

[25] J. Złotowski, E. Strasser, and C. Bartneck, "Dimensions of anthropomorphism: From humanness to humanlikeness," ACM/IEEE Int. Conf. Human-Robot Interact., pp. 66-73, 2014, doi: $10.1145 / 2559636.2559679$.

[26] V. Lim, M. Rooksby, and E. S. Cross, "Social Robots on a Global Stage: Establishing a Role for Culture During Human-Robot Interaction," Int. J. Soc. Robot., 2020, doi: 10.1007/s12369020-00710-4.

[27] P. L. P. Rau, Y. Li, and D. Li, “A cross-cultural study: Effect of robot appearance and task," Int. J. Soc. Robot., vol. 2, no. 2, pp. 175-186, 2010, doi: 10.1007/s12369-010-0056-9.

[28] M. S. Remland, T. S. Jones, and H. Brinkman, "Proxemic and haptic behavior in three European countries," J. Nonverbal Behav., vol. 15, no. 4, pp. 215-232, 1991, doi: 10.1007/BF00986923.

[29] R. E. Jack, C. Blais, C. Scheepers, P. G. Schyns, and R. Caldara, "Cultural Confusions Show that Facial Expressions Are Not Universal," Curr. Biol., vol. 19, no. 18, pp. 1543-1548, 2009, doi: 10.1016/j.cub.2009.07.051.

[30] I. Papadopoulos and C. Koulouglioti, "The Influence of Culture on Attitudes Towards Humanoid and Animal-like Robots: An Integrative Review," J. Nurs. Scholarsh., vol. 50, no. 6, pp. 653-665, 2018, doi: 10.1111/jnu.12422.

[31] D. Kuchenbrandt, F. Eyssel, S. Bobinger, and M. Neufeld, "When a Robot's Group Membership Matters: Anthropomorphization of Robots as a Function of Social Categorization," Int. J. Soc. 
Robot., vol. 5, no. 3, pp. 409-417, 2013, doi: 10.1007/s12369-013-0197-8.

431 432

433

434

435

436

437

438

439

440

441

442

443

444

445

446

447

448

449

450

451

452

453

454

455

456

[32] Z. J. Kovačić, “The impact of national culture on worldwide eGovernment readiness," Informing Sci., vol. 8, pp. 143-158, 2005, doi: 10.28945/492.

[33] J. K. Swencionis, C. H. Dupree, and S. T. Fiske, "Warmth-Competence Tradeoffs in Impression Management across Race and Social-Class Divides,” J. Soc. Issues, vol. 73, no. 1, pp. 175-191, 2017, doi: 10.1111/josi.12210.

[34] C. Bartneck, T. Suzuki, T. Kanda, and T. Nomura, "The influence of people's culture and prior experiences with Aibo on their attitude towards robots," AI Soc., vol. 21, no. 1, pp. 217-230, 2007, doi: 10.1007/s00146-006-0052-7.

[35] T. Chaminade et al., "How do we think machines think? An fMRI study of alleged competition with an artificial intelligence," Front. Hum. Neurosci., vol. 6, no. MAY 2012, pp. 1-9, 2012, doi: 10.3389/fnhum.2012.00103.

[36] H. L. Gallagher, A. I. Jack, A. Roepstorff, and C. D. Frith, "Imaging the intentional stance in a competitive game," Neuroimage, vol. 16, no. 3 I, pp. 814-821, 2002, doi: 10.1006/nimg.2002.1117.

[37] C. Özdem, E. Wiese, A. Wykowska, H. Müller, M. Brass, and F. Van Overwalle, "Believing androids-fMRI activation in the right temporo-parietal junction is modulated by ascribing intentions to non-human agents," Soc. Neurosci., vol. 12, no. 5, pp. 582-593, 2017, doi: 10.1080/17470919.2016.1207702.

[38] A. M. Abu-Akel, I. A. Apperly, S. J. Wood, and P. C. Hansen, "Re-imaging the intentional stance," Proc. R. Soc. B Biol. Sci., vol. 287, no. 1925, pp. 1-9, 2020, doi: 10.1098/rspb.2020.0244.

[39] S. Marchesi, D. Ghiglino, F. Ciardo, J. Perez-Osorio, E. Baykara, and A. Wykowska, "Do we adopt the intentional stance toward humanoid robots?," Front. Psychol., vol. 10, no. MAR, 2019, doi: 10.3389/fpsyg.2019.00450.

[40] S. Thellman, A. Silvervarg, and T. Ziemke, "Folk-psychological interpretation of human vs. humanoid robot behavior: Exploring the intentional stance toward robots," Front. Psychol., vol. 
8, no. NOV, pp. 1-14, 2017, doi: 10.3389/fpsyg.2017.01962.

458

[41] J. Perez-Osorio and A. Wykowska, "Adopting the intentional stance toward natural and artificial agents," Philos. Psychol., vol. 33, no. 3, pp. 369-395, 2020, doi: 10.1080/09515089.2019.1688778.

[42] G. Metta et al., "The iCub humanoid robot: An open-systems platform for research in cognitive development," Neural Networks, vol. 23, no. 8-9, pp. 1125-1134, 2010, doi: 10.1016/j.neunet.2010.08.010.

[43] S. Marchesi, N. Spatola, A. Wykowska, and J. Perez-Osorio, "Human vs humanoid. A behavioral investigation of the individual tendency to adopt the intentional stance," ACM/IEEE Int. Conf. Human-Robot Interact., pp. 332-340, 2021, doi: 10.1145/3434073.3444663.

[44] G. Hofstede, "Dimensionalizing Cultures: The Hofstede Model in Context," Online Readings Psychol. Cult., vol. 2, no. 1, pp. 1-26, Dec. 2011, doi: 10.9707/2307-0919.1014.

[45] B. Yoo, N. Donthu, and T. Lenartowicz, "Measuring hofstede's five dimensions of cultural values at the individual level: Development and validation of CVSCALE," J. Int. Consum. Mark., vol. 23, no. 3-4, pp. 193-210, 2011, doi: 10.1080/08961530.2011.578059.

[46] N. Spatola, B. Kühnlenz, and G. Cheng, "Perception and evaluation in human-robot interaction: The Human-Robot Interaction Evaluation Scale (HRIES) - a multicomponent approach of anthropomorphism," Int. J. Soc. Robot., 2020, doi: 10.1007/s12369-020-00667-4.

[47] N. Epley, A. Waytz, S. Akalis, and J. T. Cacioppo, "When we need a human: Motivational determinants of anthropomorphism," Soc. Cogn., vol. 26, no. 2, pp. 143-155, 2008, doi: 10.1521/soco.2008.26.2.143.

[48] N. Epley, S. Akalis, A. Waytz, and J. T. Cacioppo, "Creating social connection through inferential reproduction: Loneliness and perceived agency in gadgets, gods, and ghreyhounds," Psychol. Sci., vol. 19, no. 2, pp. 114-120, 2008, doi: 10.1111/j.1467-9280.2008.02056.x.

[49] F. Eyssel, D. Kuchenbrandt, S. Bobinger, L. De Ruiter, and F. Hegel, "'If you sound like me, you must be more human': On the interplay of robot and user features on human-robot acceptance and anthropomorphism," in HRI'12 - Proceedings of the 7th Annual ACM/IEEE 

10.1145/2157689.2157717.

486

[50] K. Lemhöfer and M. Broersma, "Introducing LexTALE: A quick and valid Lexical Test for Advanced Learners of English," Behav. Res. Methods, vol. 44, no. 2, pp. 325-343, 2012, doi: 10.3758/s13428-011-0146-0.

[51] R. Khan and P. Cox, "Country Culture and National Innovation," Arch. Bus. Res., vol. 5, no. 2, 2017, doi: 10.14738/abr.52.2768.

[52] A. L. Prim, L. S. Filho, G. A. C. Zamur, and L. C. Di Serio, "The Relationship Between National Culture Dimensions And Degree Of Innovation,” Int. J. Innov. Manag., vol. 21, no. 1, 2017, doi: 10.1142/S136391961730001X.

[53] A. Bunn and M. Korpela, "A language and environment for statistical computing," Found. Stat. Comput., vol. 2, pp. 1-12, 2013.

[54] X. Zhao, J. G. Lynch, and Q. Chen, "Reconsidering Baron and Kenny: Myths and truths about mediation analysis," J. Consum. Res., vol. 37, no. 2, pp. 197-206, 2010, doi: 10.1086/651257.

[55] S. V. T. \& R. D. H. Ulf Henning Olsson, Tron Foss, "Structural Equation Modeling: A The Performance of ML, GLS , and WLS Estimation in Structural Equation Modeling Under Conditions of Misspecification and Nonnormality," vol. 7, no. August 2012, pp. 37-41, 2009.

[56] F. Ferrari, M. P. Paladino, and J. Jetten, "Blurring Human-Machine Distinctions: Anthropomorphic Appearance in Social Robots as a Threat to Human Distinctiveness," Int. J. Soc. Robot., vol. 8, no. 2, pp. 287-302, 2016, doi: 10.1007/s12369-016-0338-y.

\section{Data Availability Statement}

The datasets for this study will be available upon acceptance in the following OSF repository [NAME OF REPOSITORY] [LINK]. 




